\title{
2009 CAEP / ACMU Scientific Abstracts Author Index
}

AUTHOR
A
Aaron S
Abu-Laban RB
Achiam CC
Adamson SA
Afilalo M
AIR Study Group
Albahrani B
Al-Dhafian A
Ali S
Aljasir B
Allegretti M
Al Mulhim K
Alrajhi A
Al-Reesi A
Al Shabanah H
Alsultan M
Alter DA
Anderson G
Andrusiek DI
Anstett D
Arishima T
Arnason T
Asahi T
Athaide V
Aubin K
Audette-Côté JS
B
Blouin D
Babyn P
Bagshaw S
Bailey B
Ball IM
Bandiera G
Banon D
Barrowman N
Bawden J
Beatty N
Ben-Yakov M
Berg RA
Bergman A
Berlin R
Bird SB
Bisset B
Blais R

\begin{tabular}{|c|c|c|}
\hline ABSTRACT No. & Boggust A & \\
\hline & Borgundvaag B & \\
\hline & Bourdeau S & \\
\hline 6,38 & Boychuk B & \\
\hline $2,6,38,48,75,142$ & Boyd E & \\
\hline 17 & Boyko D & \\
\hline 78 & Bradley K & \\
\hline $8,29,50,68,99,111$ & Brant R & \\
\hline $6,38,59,2$ & Brehaut J & \\
\hline 135 & Bretholz A & \\
\hline 166 & Brick C & \\
\hline $101,108,131$ & Brissette A & \\
\hline 34 & Bromley M & \\
\hline 137 & Brown S & \\
\hline 154 & Brubacher JR & $11,48,49,7$ \\
\hline 34 & Brulotte CA & \\
\hline 45 & Buckingham J & \\
\hline 154 & Bullard MJ & \\
\hline 34 & Butler C & \\
\hline 10 & & \\
\hline 72 & C & \\
\hline 40 & Caine $\mathrm{S}$ & \\
\hline $44,114,126,134$ & Cairney J & \\
\hline 165 & Calder L & 39 \\
\hline 129 & Campbell J & \\
\hline 165 & Campbell L & \\
\hline 155 & Campbell S & \\
\hline 153 & CanAm Pediatric & Study Grou \\
\hline 132 & Cantor WJ & \\
\hline & Carrière B & \\
\hline & Casanova A & \\
\hline 166 & Castelino $\mathrm{T}$ & \\
\hline 91 & Cembrowski G & \\
\hline $55,65,66,80$ & Chabot P & \\
\hline 79 & Chalut D & \\
\hline 93 & Chan F & \\
\hline 111 & Chan MC & \\
\hline 128 & Chan YH & \\
\hline $44,114,126$ & Charbonneau V & \\
\hline 102 & Charette $M$ & \\
\hline 148 & Chau JKM & \\
\hline 73,119 & Chen P & \\
\hline 107 & Cheng AL & \\
\hline $55,65,80$ & Cheskes S & \\
\hline 79 & Christenson J & \\
\hline 100 & Chua $\mathrm{T}$ & \\
\hline 79,132 & Clement C & \\
\hline 81 & Cohen EA & \\
\hline 22 & Colacone A & \\
\hline 5,35 & Colman I & \\
\hline
\end{tabular}

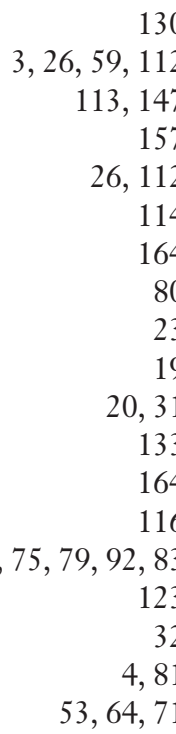

Cooke T

85,124

Copeland J

Cressman C

Crooks J

113,147

149

77,114

Cunniffe A

Cwinn A

D

Dagnone JD

Dallaire C

Dankoff J

Daoust R

Dart R

Dauphin-Pierre S

Davis MT

Dehghani N

De Maio VJ

Dionne $\mathrm{R}$

Dong KA

Dong SL

Dore KL

Dowling S

Downey L

Doyle-Waters M

Doyon-Trottier E

Ducas J

Durham C

Dyer D

Dyke L

Dzavik V

Dzwonek A

$12,21,73,78,100,119$

34,123

104

90

62

$16,28,46$

$106,116,125,162$

11

66

3

47

152

19

3

82

E

Eddleston M

Eftekhary S

Eisenhauer $M$

Émond $M$

Enarson MC

$4,24,57,120,138,139$

Eng HOM

Ernst J

Eskin B

ESSC Evaluation Group

ESSC Sub-study Evaluation Group 114

Evans J

77,114

Everson-Stewart S

Ezekowitz JA

$14,40,157$

63

143

3 F

8, 29, 68, $99 \quad$ Fan J

74,118

91 Fang $M$
79 
Fergusson D

Fernandes CMB

Filler G

Fitchett D

Foran A

Forster AJ

Foster B

Fox JL

Franc-Law JM

Frank J

Friedman S

Frush K

Fukushima E

Fung K

G

Gaebert D

Galbraith R

Gelosia A

Gill G

Glowacka K

Gnanasuntharam P

Goindi R

Golden B

Goldfarb DM

Goldman RD

Goodman SG

Grafstein E

Graham TAD

Grant GA

Gravel J

Gray SH

Green J

Green R

Grimshaw J

Guimont C

Guo R

Guttman A

Guy P

$14,25,30,52,54$, $85,124,136,157,158$

81

144

$60,61,66$

69

96

22

23

$57,68,99,132$

93

$7,29,42,27$

92

H

Hagel B

Haines C

Hall CA

Hamilton L

Hamm M

Handley M

Harris D

Harris JR

Hayter D

Healey A

Heffernan $M$

Helmich E

Hess EP

Hill DA

Hillier M

Hinchey PR

$14,52,54,136,158$

20,31

156

74,118

3

14

$3,14,16,45,46,51,86,130$

100

127

12,21
Ho JD

Ho K

Hobgood C

Hoffmann Y

Hohl CM

Hollar D

Hollohan K

Hoo YH

Houston C

Howes D

Howlett MK

$\mathrm{Hu} Z$

Hung GR

Hunte GS

Hussain Z

Hutton B

I-J

Innes GD

Ipp M

Jacob E

Jain R

Jessen KR

Jiang $\mathrm{H}$

John M

Johnson DW

Johnson J

Johnson M

Johnston T

Juurlink DN

K

Kader AS

Kamal N

Karkhaneh M

Katic BA

Kiess M

Killorn E

Kind S

Kingsley SJ

Kiss A

Kissoon N

Klassen TP

Klein-Kremer A

Knight J

Ko D

Kopetsky D

Kozan DC
Kreiswirth N

Kwok E

L

Lacasse Y

Lai A

Lali P

Lander J

Langer A
Lareau E

149 Lavoie A

37, 47 Lazzam C

18 Le B

48, 75 Leaver C

37, 47 Lee AC

14 Lee DSH

122 Lee J

77 Lee JS

133 Lee RC

70 Lee $\mathrm{S}$

11 Lehnhardt K

109 Leis S

$14,49,52,54,136,158$

LeSage N

Lesiuk $\mathrm{H}$

22 Levesque L

Lewell MP

Lewis RS

$9,14,28,30,85,115$,

$124,151,157$

Li P

Licatese D

Lim D

Lim SH

Lin D

Loewen PS

Loftus M

Loftus M Le B

Logue E

$55,65,80,108$

London

6,38

Lorette J

Louie L

Louis $M$

Lubell R

Lucas $\mathrm{N}$

111

57,138

3

112

$27,72,121$

94

79

4, 80

39

119

94

156

$57,132,139$

4

56

127,137

21, 78

10

21

146

63,122

163

48,75

112

26

101

1

22

26,112

152

76

60

M

Macintyre P 39

MacKenzie M 44

Mackey D 4, 6, 38, 59

MacLeod DB

MacNamara E

MacRedmond R

50

14

Majumdar S 6, 38, 59

39, 148

109

$6,38,58,67,97,108$

Maloney J

34,123

Manley J

13

Mansour M

87

114

Marcus R

148

Marko J

58, 67, 97

Marrie TJ

Marsden J

149

Martin CD

137

26, 112

89

Martin D

Martinez R

Martz W

Masselink WS

Maynard L

McGeer A

McIntyre L

McIsaac WJ

McLeod S
9

30

83

102

47

26, 112

22

26, 112

$1,117,155,160$ 


\begin{tabular}{|c|c|c|c|c|c|c|}
\hline McLeod SL & 17,127 & Peng HCG & 122 & Senthilselvan A & $6,38,5$ & $58,67,97$ \\
\hline Mehta S & 3,166 & Perry JJ & $4,24,41,45,51,86$ & Seviour C & & 80 \\
\hline Meiwald A & 117 & & 87,143 & Shah A & & 1,117 \\
\hline Mensour M & 74,118 & & 145 & Sharma M & & 24,87 \\
\hline Metes D & $58,67,97$ & Pirie J & 166 & Shavit I & & 18 \\
\hline Milburn S & 17 & Plint A & 80 & Sherbino J & & $2,74,118$ \\
\hline Millar K & 82 & Plourde V & 57 & Sherwood G & & 47 \\
\hline Mills B & 159 & Poitras J & 150,153 & Shouldice EH & & 145 \\
\hline Milne WK & $76,103,113,126,134$ & Porter V & 26,112 & Sibley AK & & 44 \\
\hline & $141,147,156,159$ & Poureslami I & $14,52,136,158$ & Sidhu K & & 163 \\
\hline Mithani S & 160 & Powell FA & 12,100 & Singh P & & 3 \\
\hline Moore S & 107 & Powell J & 40 & Sirois MJ & & 138 \\
\hline Morrison J & 60 & Project $\mathrm{MoVI}$ & ES Study Group $\quad 100$ & Sivilotti MLA & $2,4,24,55,65,7$ & $79,80,96$ \\
\hline Morrison LJ & 3,39 & Purssell R & $55,65,80,83$ & Slinger R & & 128 \\
\hline Mosdossy G & 1 & Pusic M & 19 & Smallfield A & & 134 \\
\hline Moylette E & 126 & & & Smith V & & 130 \\
\hline Mrklas K & 164 & $\mathbf{R}$ & & Snider C & & 15 \\
\hline Mumme S & 77 & Randall J & 31 & Snider CE & & 110 \\
\hline Munoz C & 147 & Randle E & 156 & Sock PTJ & & 122 \\
\hline Murias K & 96 & Rang L & 94 & Socransky S & & 94 \\
\hline Murphy D & 137 & Ratana $\mathrm{P}$ & 65 & Soliman AR & & 161 \\
\hline Myers JB & $21,78,119$ & Reyer E & 21 & Sookram S & & 39 \\
\hline & & Rich T & 9 & Soucy N & & $8,68,99$ \\
\hline $\mathbf{N}$ & & Rico M & 6,38 & Sowerby RJ & & 93 \\
\hline Nadkarni V & $73,78,119$ & Rieder M & 1 & Spence JM & & 69 \\
\hline Naglie G & 39 & Rodgers C & 16 & Spiers JA & & 101,131 \\
\hline Nathens A & 15 & Rose MS & 9 & Spyker D & 55,6 & $65,80,96$ \\
\hline Nebre R & 14 & Rose NGW & 92 & Stasiak P & & 8 \\
\hline Nelham M & 156 & Ross S & 2 & Steele L & & 147 \\
\hline Nestler D & 130 & Rostos A & 26,112 & Stenstrom R & $14,25,52,54,59$, & $, 136,158$ \\
\hline Nettel-Aguirre A & $55,65,80,96$ & Rosychuk RJ & $58,67,97$ & Stevens K & & 101 \\
\hline Newton AS & $\begin{array}{r}101,108 \\
108\end{array}$ & Rowe $\mathrm{BH}$ & $2,6,20,27,31,32,38,39$ & Stiell I & & $2,4,6$ \\
\hline Nichol G & 40 & & $44,58,59,67,72,77,81$ & Stiell IG & $4,16,23,24,33,3$ & $38,49,41$ \\
\hline Nichols DN & 90 & & $84,85,97,104,124,114$ & & $45,46,51,63,7$ & $73,86,87$, \\
\hline Nigam A & 121 & Roy M & 61 & & $02,119,123,129$ & 143,145 \\
\hline Noel D & 141 & Rumack B & $55,65,80,96$ & St-Onge M & & 132 \\
\hline Norman GR & 62 & Russell K & 88 & Stotts G & & 24 \\
\hline Novak-Lauscher H & 149 & Russi C & 130 & Stukel TA & & $10,42,72$ \\
\hline Nuth J & 56 & Rutledge $\mathrm{T}$ & 80 & Suan PNF & & 63 \\
\hline vutin J & 50 & Ryan DP & 39 & Sundram F & & 63 \\
\hline & & & & Sutherland J & & $4,24,87$ \\
\hline $\mathbf{O}$ & & $\mathbf{S}$ & & Svoboda T & & 26,112 \\
\hline O’Neill C & 12 & Sales A & $77,114,121$ & Swaminathan S & & 55 \\
\hline Okudera H & 165 & Salvadori $M$ & 17 & Swee HL & & 63,122 \\
\hline Olatunbosun T & 149 & Saunders LD & 84 & Sweet D & & 142 \\
\hline Osmond M & $23,73,119$ & Sawning S & 47 & Symington $\mathrm{C}$ & & 4,24 \\
\hline O’Sullivan CS & 110 & Scheuermeyer & $14,25,52,54$ & & & \\
\hline Ouston E & 34 & & $136,157,158$ & $\mathbf{T}$ & & \\
\hline & & Schlamp RS & 144 & Taddio A & & 13 \\
\hline $\mathbf{P}$ & & Schneeweiss S & 166 & Taekman J & & 47 \\
\hline Paduchowski KA & $12,78,119$ & Schoeley S & 147 & Takahashi C & & 165 \\
\hline Palmer S & $53,64,71$ & Schorn R & 85,124 & Takuhiro K & & 165 \\
\hline Patel A & 12,100 & Schreiber WE & 83 & Tam RK & & 128 \\
\hline Patterson $\mathrm{H}$ & 88 & Schull MJ 7 & $, 10,27,39,42,72,121,148$ & Taylor, A & & 49 \\
\hline Pau S & 82 & Schwartz B & 3 & Teksam O & & 140 \\
\hline Pauls M & 4 & Seabrook JA & 17 & Theakston KD & & 17 \\
\hline Payrastre J & 43 & Sedran R & 155,160 & Thiruganasamb & bandamoorthy $\mathrm{V}$ & 41,45 , \\
\hline Peddle M & 1 & Segal E & $29,50,111$ & & & 51,86 \\
\hline Peet HE & 144 & Seikaly HR & 20 & Thompson C & & 157 \\
\hline
\end{tabular}


Thompson $M$

Tillmann B

Tran CD

$55,65,79,80$

137

TRANSFER-AMI Investigators

Tredwell S

Trickett J

Tubman M

Tyberg J

U-V

Upadhye S

Vaillancourt C

Verbeek RP

Verter $\mathrm{V}$

Victorino C

Villa-Roel C

Villeneuve J

Vilneff RL

Vlahaki D

Voaklander DC

$\mathrm{Vu}$ EN

Vu MP
57,138

19

34

115

80

$43,62,74,118$

$23,33,40,46,89,135$

39

29

$55,65,96$

2, 20, 31, 32, 44, 59, 114

139

164

134

$58,67,84,97$

11,144

144
W

Waisman Y

Wakasugi $M$

Walden P

Wand RT

Wang D

Wasi $\mathrm{P}$

Webster D

Weisbrot J

Weitzman B

Wells G

Wheeler SJ

Wild TC

Willey BM

Willis V

Wilmott A

Wilson J

Wing A

Wishart I

Wisowaty S

Wong $\mathrm{H}$

Wong $\mathrm{H}$
Woo MY

Woodyard D

Worster A

Wrezel O

Wright M

Wright RB

Wuerth-Sarvis R

74,118

110

13

56

4, 16, 23, 24, 33, 41, $45,46,51,86$

144

104

26, 112

114

163

83

32,114

152

78

26

112

\section{X-Z}

Xue X

Yarascavitch A

Yarema M

Yasin A

Yeh B

Yew PNV

Ying $\mathrm{HH}$

Young B

$\mathrm{Yu}$ E

Yuen GWH

Zed PJ

Zun L

Zwarenstein M
$94,135,145$

37,47

24, 59

117

47

131

103

8, 68, 99

163

$55,65,80,96,164$

76

32

122

122

2

157

164

48,75

$106,116,125,162$ 